\title{
SOLVABILITY FOR A NONLINEAR FRACTIONAL DIFFERENTIAL EQUATION
}

\author{
YINGXIN GUO
}

(Received 11 September 2008)

\begin{abstract}
In this paper, we consider the existence of nontrivial solutions for the nonlinear fractional differential equation boundary-value problem (BVP)

$$
\begin{gathered}
\mathbf{D}^{\alpha} u(t)+f(t, u(t))+q(t)=0, \quad 0<t<1, \\
u(0)=0, \quad u(1)=\beta u(\eta)
\end{gathered}
$$

where $1<\alpha \leq 2, \eta \in(0,1), \beta \in \mathbb{R}=(-\infty,+\infty), \beta \eta^{\alpha-1} \neq 1, \mathbf{D}^{\alpha}$ is the Riemann-Liouville differential operator of order $\alpha$, and $f:[0,1] \times \mathbb{R} \rightarrow \mathbb{R}$ is continuous, $q(t):[0,1] \rightarrow[0,+\infty)$ is Lebesgue integrable. We give some sufficient conditions for the existence of nontrivial solutions to the above boundary-value problems. Our approach is based on the Leray-Schauder nonlinear alternative. Particularly, we do not use the nonnegative assumption and monotonicity on $f$ which was essential for the technique used in almost all existed literature.
\end{abstract}

2000 Mathematics subject classification: primary 34B15.

Keywords and phrases: Caputo's fractional derivative, fractional differential equation, boundary-value problem, nontrivial solution, Leray-Schauder nonlinear alternative.

\section{Introduction}

Fractional calculus has played a significant role in engineering, science, economy, and other fields. Many papers and books on fractional calculus, fractional differential equations have appeared recently, (see [1, 2, 10-12]). It should be noted that most of papers and books on fractional calculus are devoted to the solvability of linear initial fractional differential equations in terms of special functions [9]. Recently, some papers deal with the existence and multiplicity of solutions (or positive solutions) of nonlinear initial fractional differential equations by the use of techniques of nonlinear analysis (fixed-point theorems, Leray-Shauder theory, and so on), see [2, 5, 11, 12]. However, there are few papers that consider the three-point problem for linear ordinary

The authors were supported financially by the NNSF of China (10726004) and the NSF of Shandong Province of China (Q2007A02).

(c) 2009 Australian Mathematical Society 0004-9727/2009 \$16.00 
differential equations of fractional order, see $[6,8]$. No contributions exist, as far as we know, concerning the existence and multiplicity of positive solutions of the following problem:

$$
\begin{gathered}
\mathbf{D}^{\alpha} u(t)+f(t, u(t))+q(t)=0, \quad 0<t<1, \\
u(0)=0, \quad u(1)=\beta u(\eta),
\end{gathered}
$$

where $1<\alpha \leq 2, \eta \in(0,1), \beta \in \mathbb{R}=(-\infty,+\infty)$ are real numbers, $\beta \eta^{\alpha-1} \neq 1$, and $\mathbf{D}_{0+}^{\alpha}$ is the Riemann-Liouville differential operator of order $\alpha$, and $f:[0,1] \times \mathbb{R} \rightarrow \mathbb{R}$ is continuous, $q(t):[0,1] \rightarrow[0,+\infty)$ is Lebesgue integrable.

In [2], the authors consider the existence and multiplicity of positive solutions of the nonlinear fractional differential equation boundary-value problem

$$
\begin{gathered}
D_{0+}^{\alpha} u(t)+f(t, u(t))=0, \quad 0<t<1, \\
u(0)=u(1)=0
\end{gathered}
$$

where $1<\alpha \leq 2$ is a real number. $D_{0+}^{\alpha}$ is the standard Riemann-Liouville fractional derivative, and $f:[0,1] \times[0,+\infty) \rightarrow[0,+\infty)$ is continuous.

In [5], the authors consider the existence and multiplicity of positive solutions of the nonlinear fractional differential equation boundary-value problem

$$
\begin{gathered}
D^{\alpha} u(t)+a(t) f(u(t))=0, \quad 0<t<1, \\
u(0)=u^{\prime}(1)=0,
\end{gathered}
$$

where $1<\alpha \leq 2$ is a real number. $D^{\alpha}$ is the Riemann-Liouville differential operator of order $\alpha$, and $f:[0,1] \times[0,+\infty) \rightarrow[0,+\infty)$ is continuous, $a$ is a positive and continuous function on $[0,1]$.

Motivated by the work mentioned above, in this paper, we establish several sufficient conditions of the existence of nontrivial solutions for the above nonlinear fractional differential equations (1.1). Here, by a nontrivial solution of (1.1) we understand a function $u(t) \not \equiv 0$ which satisfies (1.1). Our results are new. Particularly, we do not use the nonnegative assumption and monotonicity which was essential for the technique used in almost all existing literature on $f$.

\section{Preliminaries}

For completeness, in this section, we shall demonstrate and study the definitions and some fundamental facts of fractional order.

Definition [10, Definition 2.1]. For a positive function $f(x)$ given in the interval $[0, \infty)$, the integral

$$
I^{s} f(x)=\frac{1}{\Gamma(s)} \int_{0}^{x} \frac{f(t)}{(x-t)^{1-s}} d t, \quad x>0,
$$

where $s>0$, is called the Riemann-Liouville fractional integral of order $s$. 
Definition [10, pp. 36-37]. For a positive function $f(x)$ given in the interval $[0, \infty)$, the expression

$$
D^{s} f(x)=\frac{1}{\Gamma(n-s)}\left(\frac{d}{d x}\right)^{n} \int_{0}^{x} \frac{f(t)}{(x-t)^{s-n+1}} d t,
$$

where $n=[s]+1,[s]$ denotes the integer part of number $s$, is called the RiemannLiouville fractional derivative of order $s$.

REMARK. If $f \in C[0,1] \cap L(0,1)$, then $D^{s} I^{s} f(x)=f(x)$.

In order to rewrite (1), (2) as an integral equation, we need to know the action of the fractional integral operator $I^{s}$ on $D^{s} f$ for a given function $f$. To this end, we first note that if $\lambda>-1$, then

$$
\begin{aligned}
D^{s} t^{\lambda} & =\frac{\Gamma(\lambda+1)}{\Gamma(\lambda-s+1)} t^{\lambda-s}, \\
D^{s} t^{s-k} & =0, \quad k=1,2, \ldots, n,
\end{aligned}
$$

where $n=[s]$.

The following two lemmas, found in [2], are crucial in finding an integral representation of the boundary-value problem (1.1).

LEMMA 2.1. Let $\alpha>0, u \in C[0,1] \cap L(0,1)$, then the differential equation

$$
\mathbf{D}^{\alpha} u(t)=0
$$

has solutions

$$
u(t)=c_{1} t^{\alpha-1}+c_{2} t^{\alpha-2}+\cdots+c_{n} t^{\alpha-n}, \quad c_{i} \in \mathbb{R}, i=0,1, \ldots, n, n=[\alpha]+1 .
$$

From the lemma above, we deduce the following statement.

LEMMA 2.2. Let $\alpha>0, u \in C[0,1] \cap L(0,1)$, then

$$
I^{\alpha} \mathbf{D}^{\alpha} u(t)=u(t)+c_{1} t^{\alpha-1}+c_{2} t^{\alpha-2}+\cdots+c_{n} t^{\alpha-n}
$$

for some $c_{i} \in \mathbb{R}, i=0,1, \ldots, n, n=[\alpha]+1$.

The following result will play a major role in our analysis.

LEMMA $2.3[3,7]$. Let $X$ be a real Banach space, $\Omega$ be a bounded open subset of $X, 0 \in \Omega, T: \bar{\Omega} \rightarrow X$ is a completely continuous operator. Then, either there exists $x \in \partial \Omega, \mu>1$ such that $T(x)=\mu x$, or there exists a fixed point $x^{*} \in \bar{\Omega}$.

\section{Main results}

In this section, we give our main results. First, we have the following Lemma 3.1. 
LEMMA 3.1. If $1<\alpha \leq 2, \beta \eta^{\alpha-1} \neq 1, u \in C[0,1] \cap L(0,1)$. Let $h(t) \in C[0,1]$ be a given function, then the boundary-value problem

$$
\begin{gathered}
\mathbf{D}^{\alpha} u(t)+h(t)=0, \quad 0<t<1, \\
u(0)=0, \quad u(1)=\beta u(\eta)
\end{gathered}
$$

has a unique solution

$$
\begin{gathered}
u(t)=-\frac{1}{\Gamma(\alpha)} \int_{0}^{t}(t-s)^{\alpha-1} h(s) d s+\frac{t^{\alpha-1}}{1-\beta \eta^{\alpha-1}} \frac{1}{\Gamma(\alpha)} \int_{0}^{1}(1-s)^{\alpha-1} h(s) d s \\
-\frac{\beta t^{\alpha-1}}{1-\beta \eta^{\alpha-1}} \frac{1}{\Gamma(\alpha)} \int_{0}^{\eta}(\eta-s)^{\alpha-1} h(s) d s .
\end{gathered}
$$

PROOF. By Lemma 2.2 we can reduce the equation of problem (3.1) to an equivalent integral equation

$$
u(t)=-\frac{1}{\Gamma(\alpha)} \int_{0}^{t}(t-s)^{\alpha-1} h(s) d s+c_{1} t^{\alpha-1}+c_{2} t^{\alpha-2}
$$

for some constants $c_{1}, c_{2} \in \mathbb{R}$. As boundary conditions for problem (3.1), we have $c_{2}=0$ and

$$
c_{1}=\frac{1}{1-\beta \eta^{\alpha-1}} \frac{1}{\Gamma(\alpha)}\left(\int_{0}^{1}(1-s)^{\alpha-1} h(s) d s-\beta \int_{0}^{\eta}(\eta-s)^{\alpha-1} h(s) d s\right) .
$$

Therefore, the unique solution of (3.1) is

$$
\begin{gathered}
u(t)=-\frac{1}{\Gamma(\alpha)} \int_{0}^{t}(t-s)^{\alpha-1} h(s) d s+\frac{t^{\alpha-1}}{1-\beta \eta^{\alpha-1}} \frac{1}{\Gamma(\alpha)} \int_{0}^{1}(1-s)^{\alpha-1} h(s) d s \\
-\frac{\beta t^{\alpha-1}}{1-\beta \eta^{\alpha-1}} \frac{1}{\Gamma(\alpha)} \int_{0}^{\eta}(\eta-s)^{\alpha-1} h(s) d s
\end{gathered}
$$

which completes the proof.

Let $E=C[0,1]$ be endowed with the ordering $u \leq v$ if $u(t) \leq v(t)$ for all $t \in[0,1]$, and the maximum norm, $\|u\|=\max _{0 \leq t \leq 1}|u(t)|$. Clearly, it follows that $(E,\|\cdot\|)$ is a Banach space.

Theorem 3.2. Suppose that $f(t, 0) \not \equiv 0, t \in[0,1], \beta \eta^{\alpha-1} \neq 1$, and there exist nonnegative functions $p, r \in L^{1}[0,1]$ such that

$$
\left\{\begin{array}{l}
|f(t, u)| \leq p(t)|u(t)|+r(t), \quad(t, u) \in[0,1] \times \mathbb{R}, \text { almost everywhere } \\
\frac{1}{\Gamma(\alpha)}\left[\left(1+\left|\frac{1}{1-\beta \eta}\right|\right) \int_{0}^{1}(1-s)^{\alpha-1} p(s) d s\right. \\
\left.\quad+\left|\frac{\beta}{1-\beta \eta}\right| \int_{0}^{\eta}(\eta-s)^{\alpha-1} p(s) d s\right]<1 .
\end{array}\right.
$$

Then the BVP (1.1) has at least one nontrivial solution $u^{*} \in C[0,1]$. 
PROOF. Let

$$
\begin{aligned}
A=\frac{1}{\Gamma(\alpha)} & {\left[\left(1+\left|\frac{1}{1-\beta \eta^{\alpha-1}}\right|\right) \int_{0}^{1}(1-s)^{\alpha-1} p(s) d s\right.} \\
+ & \left.\left|\frac{\beta}{1-\beta \eta^{\alpha-1}}\right| \int_{0}^{\eta}(\eta-s)^{\alpha-1} p(s) d s\right], \\
B=\frac{1}{\Gamma(\alpha)} & {\left[\left(1+\left|\frac{1}{1-\beta \eta^{\alpha-1}}\right|\right) \int_{0}^{1}(1-s)^{\alpha-1} k(s) d s\right.} \\
+ & \left.\left|\frac{\beta}{1-\beta \eta^{\alpha-1}}\right| \int_{0}^{\eta}(\eta-s)^{\alpha-1} k(s) d s\right],
\end{aligned}
$$

where $k(s)=r(s)+q(s)$. By hypothesis $A<1$. Since $f(t, 0) \not \equiv 0$, there exists $[a, b] \subset[0,1]$ such that

$$
\min _{a \leq t \leq b}|f(t, 0)|>0
$$

On the other hand, from the condition $r(t) \geq|f(t, 0)|$, almost every where $t \in[0,1]$, we know that $B>0$. Let $m=B(1-A)^{-1}, \Omega_{m}=\{u \in C[0,1]:\|u\|<m\}$.

By Lemma 3.1, problem (1.1) has a solution $u=u(t)$ if and only if $u$ solves the operator equation

$$
\begin{aligned}
(T u)(t)=-\frac{1}{\Gamma(\alpha)} & \int_{0}^{t}(t-s)^{\alpha-1}[f(s, u(s))+q(s)] d s \\
& +\frac{t^{\alpha-1}}{1-\beta \eta^{\alpha-1}} \frac{1}{\Gamma(\alpha)} \int_{0}^{1}(1-s)^{\alpha-1}[f(s, u(s))+q(s)] d s \\
& -\frac{\beta t^{\alpha-1}}{1-\beta \eta^{\alpha-1}} \frac{1}{\Gamma(\alpha)} \int_{0}^{\eta}(\eta-s)^{\alpha-1}[f(s, u(s))+q(s)] d s
\end{aligned}
$$

in $E$. So we only need to seek a fixed point of $T$ in $E$. By the Ascoli-Arzela theorem, it is well known that this operator $T: E \rightarrow E$ is a completely continuous operator.

Suppose $u \in \partial \Omega_{m}, \mu>1$ such that $T u=\mu u$; then

$$
\begin{aligned}
\mu m=\mu\|u\| & =\|T u\|=\max _{0 \leq t \leq 1}|(T u)(t)| \\
\leq \max _{0 \leq t \leq 1} & \frac{1}{\Gamma(\alpha)} \int_{0}^{t}(t-s)^{\alpha-1}|f(s, u(s))+q(s)| d s \\
& +\max _{0 \leq t \leq 1} \frac{t^{\alpha-1}}{\left|1-\beta \eta^{\alpha-1}\right|} \frac{1}{\Gamma(\alpha)} \int_{0}^{1}(1-s)^{\alpha-1}|f(s, u(s))+q(s)| d s \\
& +\max _{0 \leq t \leq 1} \frac{|\beta| t^{\alpha-1}}{\left|1-\beta \eta^{\alpha-1}\right|} \frac{1}{\Gamma(\alpha)} \int_{0}^{\eta}(\eta-s)^{\alpha-1}|f(s, u(s))+q(s)| d s \\
\leq \frac{1}{\Gamma(\alpha)} & \int_{0}^{1}(1-s)^{\alpha-1}(|f(s, u(s))|+q(s)) d s \\
& +\frac{1}{\left|1-\beta \eta^{\alpha-1}\right|} \frac{1}{\Gamma(\alpha)} \int_{0}^{1}(1-s)^{\alpha-1}(|f(s, u(s))|+q(s)) d s
\end{aligned}
$$




$$
\begin{aligned}
+ & \left|\frac{\beta}{1-\beta \eta^{\alpha-1}}\right| \frac{1}{\Gamma(\alpha)} \int_{0}^{\eta}(\eta-s)^{\alpha-1}(|f(s, u(s))|+q(s)) d s \\
\leq(1+ & \left.\left|\frac{1}{1-\beta \eta^{\alpha-1}}\right|\right) \frac{1}{\Gamma(\alpha)} \int_{0}^{1}(1-s)^{\alpha-1}[p(s)|u(s)|+r(s)+q(s)] d s \\
+ & \frac{\beta}{1-\beta \eta^{\alpha-1}} \mid \frac{1}{\Gamma(\alpha)} \int_{0}^{\eta}(\eta-s)^{\alpha-1}[p(s)|u(s)|+r(s)+q(s)] d s \\
\leq \frac{1}{\Gamma(\alpha)} & {\left[\left(1+\left|s \frac{1}{1-\beta \eta^{\alpha-1}}\right|\right) \int_{0}^{1}(1-s)^{\alpha-1} p(s) d s\right.} \\
+ & \left.\left|\frac{\beta}{1-\beta \eta^{\alpha-1}}\right| \int_{0}^{\eta}(\eta-s)^{\alpha-1} p(s) d s\right]\|u\| \\
+ & \frac{1}{\Gamma(\alpha)}\left[\left(1+\left|\frac{1}{1-\beta \eta^{\alpha-1}}\right|\right) \int_{0}^{1}(1-s)^{\alpha-1}[r(s)+q(s)] d s\right. \\
+ & \left.\left|\frac{\beta}{1-\beta \eta^{\alpha-1}}\right| \int_{0}^{\eta}(\eta-s)^{\alpha-1}[r(s)+q(s)] d s\right] \\
\leq A\|u\| & +B=A m+B .
\end{aligned}
$$

Therefore

$$
\mu \leq A+\frac{B}{m}=A+\frac{B}{B(1-A)^{-1}}=A+(1-A)=1 .
$$

This contradicts $\mu>1$. By Lemma $2.4, T$ has a fixed point $u^{*} \in \bar{\Omega}$. Since $f(t, 0) \not \equiv 0$, the BVP (1.1) has a nontrivial solution $u^{*} \in C[0,1]$. This completes the proof.

TheOREM 3.3. Suppose that $f(t, 0) \not \equiv 0, t \in[0,1], \beta \eta^{\alpha-1}<1$, and there exist nonnegative functions $p, r \in L^{1}[0,1]$ such that

$$
|f(t, u)| \leq p(t)|u(t)|+r(t), \quad(t, u) \in[0,1] \times \mathbb{R}, \text { almost everywhere }
$$

and one of the following conditions holds.

(1) There exists a constant $\lambda>1$ such that

$$
\int_{0}^{1} p(s)^{\lambda} d s<\left[\frac{\Gamma(\alpha)\left(1-\beta \eta^{\alpha-1}\right)[1+\kappa(\alpha-1)]^{1 / \kappa}}{2-\beta \eta^{\alpha-1}+|\beta| \eta^{1 / \kappa}}\right]^{\lambda} \quad\left(\frac{1}{\lambda}+\frac{1}{\kappa}=1\right) .
$$

(2) The function $p(s)$ satisfies

$$
\left\{\begin{array}{l}
p(s) \leq \frac{\Gamma(\alpha) \alpha\left(1-\beta \eta^{\alpha-1}\right)}{2-\beta \eta^{\alpha-1}+|\beta| \eta^{\alpha}}, \quad s \in[0,1], \text { almost everywhere } \\
\operatorname{mes}\left\{s \in[0,1] ; p(s)<\frac{\Gamma(\alpha) \alpha\left(1-\beta \eta^{\alpha-1}\right)}{2-\beta \eta^{\alpha-1}+|\beta| \eta^{\alpha}}\right\}>0 .
\end{array}\right.
$$


(3) There exists a constant $\mu>-\alpha$ such that

$$
\left\{\begin{array}{l}
p(s) \leq \frac{\Gamma(\alpha)(\alpha+\mu)\left(1-\beta \eta^{\alpha-1}\right)}{2-\beta \eta^{\alpha-1}+|\beta|\left[1-(1-\eta)^{\alpha+\mu}\right]}(1-s)^{\mu}, \\
s \in[0,1], \text { almost everywhere } \\
\operatorname{mes}\left\{s \in[0,1] ; p(s)<\frac{\Gamma(\alpha)(\alpha+\mu)\left(1-\beta \eta^{\alpha-1}\right)}{2-\beta \eta^{\alpha-1}+|\beta|\left[1-(1-\eta)^{\alpha+\mu}\right]}(1-s)^{\mu}\right\}>0 .
\end{array}\right.
$$

(4) There exists a constant $\mu>-1$ such that

$$
\left\{\begin{array}{l}
p(s) \leq \frac{\Gamma(\alpha)(1+\mu)\left(1-\beta \eta^{\alpha-1}\right)}{2-\beta \eta^{\alpha-1}+|\beta| \eta^{1+\mu}} \frac{s^{\mu}}{(1-s)^{\alpha-1}}, \\
\quad s \in[0,1], \text { almost everywhere } \\
\operatorname{mes}\left\{s \in[0,1] ; p(s)<\frac{\Gamma(\alpha)(1+\mu)\left(1-\beta \eta^{\alpha-1}\right)}{2-\beta \eta^{\alpha-1}+|\beta| \eta^{1+\mu}} \frac{s^{\mu}}{(1-s)^{\alpha-1}}\right\}>0 .
\end{array}\right.
$$

Then the BVP (1.1) has at least one nontrivial solution $u^{*} \in C[0,1]$.

Proof. Let $A$ be as in Theorem 3.2, we only need to prove $A<1$. Note that $\beta \eta^{\alpha-1}<1$. We have the following cases.

(1) In this case, by using the Hölder inequality,

$$
\begin{aligned}
A & =\frac{1}{\Gamma(\alpha)}\left[\left(1+\left|\frac{1}{1-\beta \eta^{\alpha-1}}\right|\right) \int_{0}^{1}(1-s)^{\alpha-1} p(s) d s\right. \\
& \left.+\left|\frac{\beta}{1-\beta \eta^{\alpha-1}}\right| \int_{0}^{\eta}(\eta-s)^{\alpha-1} p(s) d s\right] \\
& \leq \frac{1}{\Gamma(\alpha)}\left[\int_{0}^{1} p(s)^{\lambda} d s\right]^{1 / \lambda}\left\{\frac{2-\beta \eta^{\alpha-1}}{1-\beta \eta^{\alpha-1}}\left[\int_{0}^{1}(1-s)^{\kappa(\alpha-1)} d s\right]^{1 / \kappa}\right. \\
& +\frac{|\beta|}{1-\beta \eta^{\alpha-1}}\left[\int _ { 0 } ^ { \eta } \frac { ( \eta - s ) ^ { \kappa ( \alpha - 1 ) } d s ] ^ { 1 / \kappa } \} } { \Gamma ( \alpha ) } [ \int _ { 0 } ^ { 1 } p ( s ) ^ { \lambda } d s ] ^ { 1 / \lambda } \left\{\frac{2-\beta \eta^{\alpha-1}}{1-\beta \eta^{\alpha-1}}\left[\frac{1}{1+\kappa(\alpha-1)}\right]^{1 / \kappa}\right.\right. \\
& \left.+\frac{|\beta|}{1-\beta \eta^{\alpha-1}}\left[\frac{\eta^{1+\kappa(\alpha-1)}}{1+\kappa(\alpha-1)}\right]^{1 / \kappa}\right\} \\
& \leq \frac{1}{\Gamma(\alpha)}\left[\int_{0}^{1} p(s)^{\lambda} d s\right]^{1 / \lambda} \frac{2-\beta \eta^{\alpha-1}+|\beta| \eta^{1 / \kappa}}{\left(1-\beta \eta^{\alpha-1}\right)[1+\kappa(\alpha-1)]^{1 / \kappa}} \\
& <\frac{1}{\Gamma(\alpha)} \cdot \frac{\Gamma(\alpha)\left(1-\beta \eta^{\alpha-1}\right)[1+\kappa(\alpha-1)]^{1 / \kappa}}{2-\beta \eta^{\alpha-1}+|\beta| \eta^{1 / \kappa}} \frac{2-\beta \eta^{\alpha-1}+|\beta| \eta^{1 / \kappa}}{\left(1-\beta \eta^{\alpha-1}\right)[1+\kappa(\alpha-1)]^{1 / \kappa}} \\
& =1 .
\end{aligned}
$$


(2) In this case,

$$
\begin{aligned}
A<\frac{1}{\Gamma(\alpha)} & \frac{\Gamma(\alpha) \alpha\left(1-\beta \eta^{\alpha-1}\right)}{2-\beta \eta^{\alpha-1}+|\beta| \eta^{\alpha}}\left[\frac{2-\beta \eta^{\alpha-1}}{1-\beta \eta^{\alpha-1}} \int_{0}^{1}(1-s)^{\alpha-1} d s\right. \\
& \left.\quad+\frac{|\beta|}{1-\beta \eta^{\alpha-1}} \int_{0}^{\eta}(\eta-s)^{\alpha-1} d s\right] \\
= & \frac{1}{\Gamma(\alpha)} \cdot \frac{\Gamma(\alpha) \alpha\left(1-\beta \eta^{\alpha-1}\right)}{2-\beta \eta^{\alpha-1}+|\beta| \eta^{\alpha}} \cdot \frac{2-\beta \eta^{\alpha-1}+|\beta| \eta^{\alpha}}{\alpha\left(1-\beta \eta^{\alpha-1}\right)}=1 .
\end{aligned}
$$

(3) In this case,

$$
\begin{aligned}
A<\frac{1}{\Gamma(\alpha)} & {\left[\frac{2-\beta \eta^{\alpha-1}}{1-\beta \eta^{\alpha-1}} \int_{0}^{1}(1-s)^{\alpha-1}(1-s)^{\mu} d s\right.} \\
& \left.+\frac{|\beta|}{1-\beta \eta^{\alpha-1}} \int_{0}^{\eta}(\eta-s)^{\alpha-1}(1-s)^{\mu} d s\right] \\
& \times \frac{\Gamma(\alpha)(\alpha+\mu)\left(1-\beta \eta^{\alpha-1}\right)}{2-\beta \eta^{\alpha-1}+|\beta|\left[1-(1-\eta)^{\alpha+\mu}\right]} \\
<\frac{1}{\Gamma(\alpha)} & {\left[\frac{2-\beta \eta^{\alpha-1}}{1-\beta \eta^{\alpha-1}} \int_{0}^{1}(1-s)^{\alpha-1}(1-s)^{\mu} d s\right.} \\
+ & \left.\frac{|\beta|}{1-\beta \eta^{\alpha-1}} \int_{0}^{\eta}(1-s)^{\alpha-1}(1-s)^{\mu} d s\right] \\
\times & \times \frac{\Gamma(\alpha)(\alpha+\mu)\left(1-\beta \eta^{\alpha-1}\right)}{2-\beta \eta^{\alpha-1}+|\beta|\left[1-(1-\eta)^{\alpha+\mu}\right]} \\
=\frac{1}{\Gamma(\alpha)} & {\left[\frac{2-\beta \eta^{\alpha-1}}{1-\beta \eta^{\alpha-1}} \frac{1}{\alpha+\mu}+\frac{|\beta|}{1-\beta \eta^{\alpha-1}} \frac{1-(1-\eta)^{\alpha+\mu}}{\alpha+\mu}\right] } \\
\times & \times \frac{\Gamma(\alpha)(\alpha+\mu)\left(1-\beta \eta^{\alpha-1}\right)}{2-\beta \eta^{\alpha-1}+|\beta|\left[1-(1-\eta)^{\alpha+\mu}\right]} \\
=1 . &
\end{aligned}
$$

(4) In this case,

$$
\begin{aligned}
A<\frac{1}{\Gamma(\alpha)} & {\left[\frac{2-\beta \eta^{\alpha-1}}{1-\beta \eta^{\alpha-1}} \int_{0}^{1}(1-s)^{\alpha-1} \frac{s^{\mu}}{(1-s)^{\alpha-1}} d s\right.} \\
& \left.+\frac{|\beta|}{1-\beta \eta^{\alpha-1}} \int_{0}^{\eta}(\eta-s)^{\alpha-1} \frac{s^{\mu}}{(1-s)^{\alpha-1}} d s\right] \cdot \frac{\Gamma(\alpha)(1+\mu)\left(1-\beta \eta^{\alpha-1}\right)}{2-\beta \eta^{\alpha-1}+|\beta| \eta^{1+\mu}} \\
<\frac{1}{\Gamma(\alpha)} & {\left[\frac{2-\beta \eta^{\alpha-1}}{1-\beta \eta^{\alpha-1}} \int_{0}^{1} s^{\mu} d s+\frac{|\beta|}{1-\beta \eta^{\alpha-1}} \int_{0}^{\eta}(1-s)^{\alpha-1} \frac{s^{\mu}}{(1-s)^{\alpha-1}} d s\right] } \\
& \times \frac{\Gamma(\alpha)(1+\mu)\left(1-\beta \eta^{\alpha-1}\right)}{2-\beta \eta^{\alpha-1}+|\beta| \eta^{1+\mu}}
\end{aligned}
$$




$$
\begin{aligned}
& =\frac{1}{\Gamma(\alpha)}\left[\frac{2-\beta \eta^{\alpha-1}}{1-\beta \eta^{\alpha-1}} \frac{1}{1+\mu}+\frac{|\beta|}{1-\beta \eta^{\alpha-1}} \frac{\eta^{1+\mu}}{1+\mu}\right] \cdot \frac{\Gamma(\alpha)(1+\mu)\left(1-\beta \eta^{\alpha-1}\right)}{2-\beta \eta^{\alpha-1}+|\beta| \eta^{1+\mu}} \\
& =1 .
\end{aligned}
$$

Then, from Theorem 3.2, we know the BVP (1.1) has at least one nontrivial solution $u^{*} \in C[0,1]$.

THEOREM 3.4. Suppose that $f(t, 0) \not \equiv 0, t \in[0,1], \beta \eta^{\alpha-1}>1$, and there exist nonnegative functions $p, r \in L^{1}[0,1]$ such that

$$
|f(t, u)| \leq p(t)|u(t)|+r(t), \quad(t, u) \in[0,1] \times \mathbb{R}, \text { almost everywhere }
$$

and one of the following conditions holds.

(1) There exists a constant $\lambda>1$ such that

$$
\int_{0}^{1} p(s)^{\lambda} d s<\left[\frac{\Gamma(\alpha)\left(\beta \eta^{\alpha-1}-1\right)[1+\kappa(\alpha-1)]^{1 / \kappa}}{\beta\left(\eta^{\alpha-1}+\eta^{1 / \kappa}\right)}\right]^{\lambda} \quad\left(\frac{1}{\lambda}+\frac{1}{\kappa}=1\right) .
$$

(2) The function $p(s)$ satisfies

$$
\left\{\begin{array}{l}
p(s) \leq \frac{\Gamma(\alpha) \alpha\left(\beta \eta^{\alpha-1}-1\right)}{\beta\left(\eta^{\alpha-1}+\eta^{\alpha}\right)}, \quad s \in[0,1], \text { almost everywhere } \\
\operatorname{mes}\left\{s \in[0,1] ; p(s)<\frac{\Gamma(\alpha) \alpha\left(\beta \eta^{\alpha-1}-1\right)}{\beta\left(\eta^{\alpha-1}+\eta^{\alpha}\right)}\right\}>0 .
\end{array}\right.
$$

(3) There exists a constant $\mu>-\alpha$ such that

$$
\left\{\begin{array}{l}
p(s) \leq \frac{\Gamma(\alpha)(\alpha+\mu)\left(\beta \eta^{\alpha-1}-1\right)}{\beta\left[\eta^{\alpha-1}+1-(1-\eta)^{\alpha+\mu}\right]}(1-s)^{\mu}, \\
s \in[0,1], \text { almost everywhere } \\
\operatorname{mes}\left\{s \in[0,1] ; p(s)<\frac{\Gamma(\alpha)(\alpha+\mu)\left(\beta \eta^{\alpha-1}-1\right)}{\beta\left[\eta^{\alpha-1}+1-(1-\eta)^{\alpha+\mu}\right]}(1-s)^{\mu}\right\}>0 .
\end{array}\right.
$$

(4) There exists a constant $\mu>-1$ such that

$$
\left\{\begin{array}{l}
p(s) \leq \frac{\Gamma(\alpha)(1+\mu)\left(\beta \eta^{\alpha-1}-1\right)}{\beta\left(\eta^{\alpha-1}+\eta^{1+\mu}\right)} \frac{s^{\mu}}{(1-s)^{\alpha-1}}, \\
s \in[0,1], \text { almost everywhere } \\
\operatorname{mes}\left\{s \in[0,1] ; p(s)<\frac{\Gamma(\alpha)(1+\mu)\left(\beta \eta^{\alpha-1}-1\right)}{\beta\left(\eta^{\alpha-1}+\eta^{1+\mu}\right)} \frac{s^{\mu}}{(1-s)^{\alpha-1}}\right\}>0 .
\end{array}\right.
$$

Then the BVP (1.1) has at least one nontrivial solution $u^{*} \in C[0,1]$. 
Proof. Let $A$ be as in Theorem 3.2, we only need to prove $A<1$. Note that $\beta \eta^{\alpha-1}>1$. We have the following cases.

(1) In this case, by using the Hölder inequality,

$$
\begin{aligned}
A & =\frac{1}{\Gamma(\alpha)}\left[\left(1+\left|\frac{1}{1-\beta \eta^{\alpha-1}}\right|\right) \int_{0}^{1}(1-s)^{\alpha-1} p(s) d s\right. \\
& \left.+\left|\frac{\beta}{1-\beta \eta^{\alpha-1}}\right| \int_{0}^{\eta}(\eta-s)^{\alpha-1} p(s) d s\right] \\
& \leq \frac{1}{\Gamma(\alpha)}\left[\int_{0}^{1} p(s)^{\lambda} d s\right]^{1 / \lambda}\left\{\frac{\beta \eta^{\alpha-1}}{\beta \eta^{\alpha-1}-1}\left[\int_{0}^{1}(1-s)^{\kappa(\alpha-1)} d s\right]^{1 / \kappa}\right. \\
& +\frac{\beta}{\beta \eta^{\alpha-1}-1}\left[\int _ { 0 } ^ { \eta } \frac { ( \eta - s ) ^ { \kappa ( \alpha - 1 ) } d s ] ^ { 1 / \kappa } \} } { \Gamma ( \alpha ) } [ \int _ { 0 } ^ { 1 } p ( s ) ^ { \lambda } d s ] ^ { 1 / \lambda } \left\{\frac{\beta \eta^{\alpha-1}}{\beta \eta^{\alpha-1}-1}\left[\frac{1}{1+\kappa(\alpha-1)}\right]^{1 / \kappa}\right.\right. \\
& \left.+\frac{\beta}{\beta \eta^{\alpha-1}-1}\left[\frac{\eta^{1+\kappa(\alpha-1)}}{1+\kappa(\alpha-1)}\right]^{1 / \kappa}\right\} \\
& \leq \frac{1}{\Gamma(\alpha)}\left[\int_{0}^{1} p(s)^{\lambda} d s\right]^{1 / \lambda} \frac{\beta \eta^{\alpha-1}+\beta \eta^{1 / \kappa}}{\left(\beta \eta^{\alpha-1}-1\right)[1+\kappa(\alpha-1)]^{1 / \kappa}} \\
& <\frac{1}{\Gamma(\alpha)} \cdot \frac{\Gamma(\alpha)\left(\beta \eta^{\alpha-1}-1\right)[1+\kappa(\alpha-1)]^{1 / \kappa}}{\beta\left(\eta^{\alpha-1}+\eta^{1 / \kappa}\right)} \cdot \frac{\beta\left(\eta^{\alpha-1}+\eta^{1 / \kappa}\right)}{\left(\beta \eta^{\alpha-1}-1\right)[1+\kappa(\alpha-1)]^{1 / \kappa}} \\
& =1 .
\end{aligned}
$$

(2) In this case,

$$
\begin{aligned}
A< & \frac{1}{\Gamma(\alpha)} \frac{\Gamma(\alpha) \alpha\left(\beta \eta^{\alpha-1}-1\right)}{\beta\left(\eta^{\alpha-1}+\eta^{\alpha}\right)}\left[\frac{\beta \eta^{\alpha-1}}{\beta \eta^{\alpha-1}-1} \int_{0}^{1}(1-s)^{\alpha-1} d s\right. \\
& \left.+\frac{\beta}{\beta \eta^{\alpha-1}-1} \int_{0}^{\eta}(\eta-s)^{\alpha-1} d s\right] \\
= & \frac{1}{\Gamma(\alpha)} \cdot \frac{\Gamma(\alpha) \alpha\left(\beta \eta^{\alpha-1}-1\right)}{\beta\left(\eta^{\alpha-1}+\eta^{\alpha}\right)} \cdot \frac{\beta\left(\eta^{\alpha-1}+\eta^{\alpha}\right)}{\alpha\left(\beta \eta^{\alpha-1}-1\right)}=1 .
\end{aligned}
$$

(3) In this case,

$$
\begin{aligned}
A<\frac{1}{\Gamma(\alpha)} & {\left[\frac{\beta \eta^{\alpha-1}}{\beta \eta^{\alpha-1}-1} \int_{0}^{1}(1-s)^{\alpha-1}(1-s)^{\mu} d s\right.} \\
& \left.+\frac{\beta}{\beta \eta^{\alpha-1}-1} \int_{0}^{\eta}(\eta-s)^{\alpha-1}(1-s)^{\mu} d s\right] \cdot \frac{\Gamma(\alpha)(\alpha+\mu)\left(\beta \eta^{\alpha-1}-1\right)}{\beta\left[\eta^{\alpha-1}+1-(1-\eta)^{\alpha+\mu}\right]}
\end{aligned}
$$




$$
\begin{aligned}
& <\frac{1}{\Gamma(\alpha)}\left[\frac{\beta \eta^{\alpha-1}}{\beta \eta^{\alpha-1}-1} \int_{0}^{1}(1-s)^{\alpha-1}(1-s)^{\mu} d s\right. \\
& \left.+\frac{\beta}{\beta \eta^{\alpha-1}-1} \int_{0}^{\eta}(1-s)^{\alpha-1}(1-s)^{\mu} d s\right] \cdot \frac{\Gamma(\alpha)(\alpha+\mu)\left(\beta \eta^{\alpha-1}-1\right)}{\beta\left[\eta^{\alpha-1}+1-(1-\eta)^{\alpha+\mu}\right]} \\
& =\frac{1}{\Gamma(\alpha)}\left[\frac{\beta \eta^{\alpha-1}}{\beta \eta^{\alpha-1}-1} \frac{1}{\alpha+\mu}+\frac{\beta}{\beta \eta^{\alpha-1}-1} \frac{1-(1-\eta)^{\alpha+\mu}}{\alpha+\mu}\right] \\
& \quad \times \frac{\Gamma(\alpha)(\alpha+\mu)\left(\beta \eta^{\alpha-1}-1\right)}{\beta\left[\eta^{\alpha-1}+1-(1-\eta)^{\alpha+\mu}\right]} \\
& =1 .
\end{aligned}
$$

(4) In this case,

$$
\begin{aligned}
A< & \frac{1}{\Gamma(\alpha)}\left[\frac{\beta \eta^{\alpha-1}}{\beta \eta^{\alpha-1}-1} \int_{0}^{1}(1-s)^{\alpha-1} \frac{s^{\mu}}{(1-s)^{\alpha-1}} d s\right. \\
& \left.+\frac{\beta}{\beta \eta^{\alpha-1}-1} \int_{0}^{\eta}(\eta-s)^{\alpha-1} \frac{s^{\mu}}{(1-s)^{\alpha-1}} d s\right] \cdot \frac{\Gamma(\alpha)(1+\mu)\left(\beta \eta^{\alpha-1}-1\right)}{\beta\left(\eta^{\alpha-1}+\eta^{1+\mu}\right)} \\
< & \frac{1}{\Gamma(\alpha)}\left[\frac{\beta \eta^{\alpha-1}}{\beta \eta^{\alpha-1}-1} \int_{0}^{1} s^{\mu} d s+\frac{\beta}{\beta \eta^{\alpha-1}-1} \int_{0}^{\eta}(1-s)^{\alpha-1} \frac{s^{\mu}}{(1-s)^{\alpha-1}} d s\right] \\
& \times \frac{\Gamma(\alpha)(1+\mu)\left(\beta \eta^{\alpha-1}-1\right)}{\beta\left(\eta^{\alpha-1}+\eta^{1+\mu}\right)} \\
= & \frac{1}{\Gamma(\alpha)}\left[\frac{\beta \eta^{\alpha-1}}{\beta \eta^{\alpha-1}-1} \frac{1}{1+\mu}+\frac{\beta}{\beta \eta^{\alpha-1}-1} \frac{\eta^{1+\mu}}{1+\mu}\right] \cdot \frac{\Gamma(\alpha)(1+\mu)\left(\beta \eta^{\alpha-1}-1\right)}{\beta\left(\eta^{\alpha-1}+\eta^{1+\mu}\right)} \\
= & 1 .
\end{aligned}
$$

Then, from Theorem 3.2, we know the BVP (1.1) has at least one nontrivial solution $u^{*} \in C[0,1]$.

THEOREM 3.5. Suppose that $f(t, 0) \not \equiv 0$, and there exist nonnegative functions $p \in$ $L^{1}[0,1]$ such that

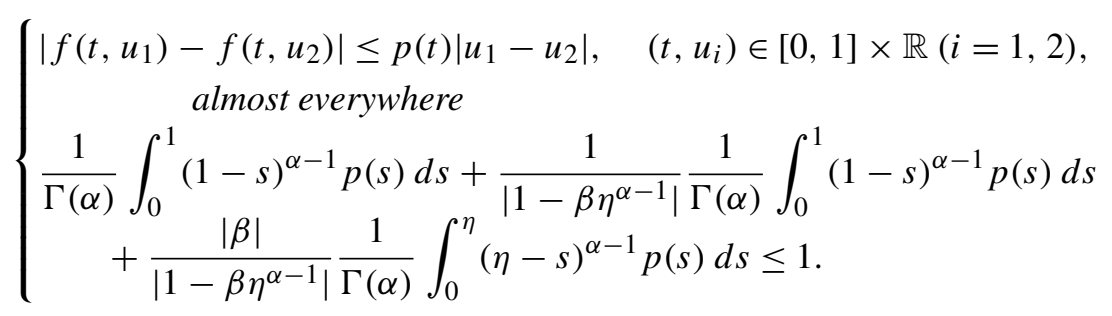

Then the BVP (1.1) has at least one nontrivial solution $u^{*} \in C[0,1]$.

PRoOF. In fact, if $u_{2}=0$, then we have $\left|f\left(t, u_{1}\right)\right| \leq p(t)\left|u_{1}\right|+|f(t, 0)|,\left(t, u_{1}\right) \in$ $[0,1] \times \mathbb{R}$ almost everywhere. From Theorem 3.1, we know the BVP (1.1) has a nontrivial solution $u^{*} \in C^{1}[0,1]$. 
But in this case, we prefer to concentrate on the uniqueness of nontrivial solutions for the BVP (1.1). Let $T$ be given in Theorem 3.2, we shall show that $T$ is a contraction. In fact,

$$
\begin{aligned}
& \left\|T u_{1}-T u_{2}\right\| \\
& =\max _{0 \leq t \leq 1}\left|\left(T u_{1}\right)(t)-\left(T u_{2}\right)(t)\right| \\
& \leq \max _{0 \leq t \leq 1} \frac{1}{\Gamma(\alpha)} \int_{0}^{t}(t-s)^{\alpha-1}\left|f\left(s, u_{1}(s)\right)-f\left(s, u_{2}(s)\right)\right| d s \\
& +\max _{0 \leq t \leq 1} \frac{t}{\left|1-\beta \eta^{\alpha-1}\right|} \frac{1}{\Gamma(\alpha)} \int_{0}^{1}(1-s)^{\alpha-1}\left|f\left(s, u_{1}(s)\right)-f\left(s, u_{2}(s)\right)\right| d s \\
& +\max _{0 \leq t \leq 1} \frac{|\beta| t}{\left|1-\beta \eta^{\alpha-1}\right|} \frac{1}{\Gamma(\alpha)} \int_{0}^{\eta}(\eta-s)^{\alpha-1}\left|f\left(s, u_{1}(s)\right)-f\left(s, u_{2}(s)\right)\right| d s \\
& \leq \max _{0 \leq t \leq 1} \frac{1}{\Gamma(\alpha)} \int_{0}^{t}(t-s)^{\alpha-1} p(s)\left|u_{1}-u_{2}\right| d s \\
& +\max _{0 \leq t \leq 1} \frac{t}{\left|1-\beta \eta^{\alpha-1}\right|} \frac{1}{\Gamma(\alpha)} \int_{0}^{1}(1-s)^{\alpha-1} p(s)\left|u_{1}-u_{2}\right| d s \\
& +\max _{0 \leq t \leq 1} \frac{|\beta| t}{\left|1-\beta \eta^{\alpha-1}\right|} \frac{1}{\Gamma(\alpha)} \int_{0}^{\eta}(\eta-s)^{\alpha-1} p(s)\left|u_{1}-u_{2}\right| d s \\
& \leq \frac{1}{\Gamma(\alpha)} \int_{0}^{1}(1-s)^{\alpha-1} p(s)\left|u_{1}-u_{2}\right| d s \\
& +\frac{1}{\left|1-\beta \eta^{\alpha-1}\right|} \frac{1}{\Gamma(\alpha)} \int_{0}^{1}(1-s)^{\alpha-1} p(s)\left|u_{1}-u_{2}\right| d s \\
& +\frac{|\beta|}{\left|1-\beta \eta^{\alpha-1}\right|} \frac{1}{\Gamma(\alpha)} \int_{0}^{\eta}(\eta-s)^{\alpha-1} p(s)\left|u_{1}-u_{2}\right| d s \\
& \leq\left[\frac{1}{\Gamma(\alpha)} \int_{0}^{1}(1-s)^{\alpha-1} p(s) d s+\frac{1}{\left|1-\beta \eta^{\alpha-1}\right|} \frac{1}{\Gamma(\alpha)} \int_{0}^{1}(1-s)^{\alpha-1} p(s) d s\right. \\
& \left.+\frac{|\beta|}{\left|1-\beta \eta^{\alpha-1}\right|} \frac{1}{\Gamma(\alpha)} \int_{0}^{\eta}(\eta-s)^{\alpha-1} p(s) d s\right]\left\|u_{1}-u_{2}\right\| \\
& \leq\left\|u_{1}-u_{2}\right\| \text {. }
\end{aligned}
$$

So $T$ is indeed a contraction. Finally we use the Banach fixed point theorem to deduce the existence of a unique solution to the BVP (1.1).

COROLlary 3.6. Suppose that $f(t, 0) \neq 0$, and

$$
\left\{\begin{array}{l}
0 \leq M=\lim \sup _{|u| \rightarrow+\infty} \max _{0 \leq t \leq 1} \frac{|f(t, u)|}{|u|}<+\infty, \\
\frac{M+1-\varepsilon}{\alpha \Gamma(\alpha)}\left[1+\frac{1}{\left|1-\beta \eta^{\alpha-1}\right|}+\frac{|\beta| \eta^{\alpha}}{\left|1-\beta \eta^{\alpha-1}\right|}\right] \leq 1
\end{array}\right.
$$


where $\varepsilon>0$ such that $M+1-\varepsilon>0$. Then (1.1) has at least one nontrivial solution $u^{*} \in C[0,1]$.

ProOf. Let $\varepsilon>0$ such that $M+1-\varepsilon>0$. By (*), there exists $H>0$ such that

$$
|f(t, u)| \leq(M+1-\varepsilon)|u|, \quad|u| \geq H, 0 \leq t \leq 1 .
$$

Let $N=\max _{t \in[0,1],|u| \leq H}|f(t, u)|$. Then for any $(t, u) \in[0,1] \times \mathbb{R}$, we have

$$
|f(t, u)| \leq(M+1-\varepsilon)|u|+N .
$$

From Theorem 3.5 we know the $B V P(1.1)$ has at least one nontrivial solution $u^{*} \in C[0,1]$.

\section{Examples}

EXAMPLE 4.1. Consider the following third-order three-point problem:

$$
\left\{\begin{array}{l}
\mathbf{D}^{3 / 2} y(t)=y \frac{3 t^{2} \sin t}{2 \sqrt{1-t}}+t^{3}+\cos t, \quad 0<t<1, \\
y(0)=0, \quad y(1)=\beta y\left(\frac{1}{2}\right),
\end{array}\right.
$$

where $\beta>34 \sqrt{2} / 9, f(t, y)=y 3 t^{2} \sin t / 2 \sqrt{1-t}+t^{3}$. We choose $p(t)=3 t^{2} /$ $2 \sqrt{1-t}, r(t)=t^{3}$, then

$$
\begin{aligned}
A= & \frac{1}{\Gamma(3 / 2)}\left[\left(1+\frac{\sqrt{2}}{\beta-\sqrt{2}}\right) \int_{0}^{1} \sqrt{1-s} \frac{3 s^{2}}{2 \sqrt{1-s}} d s\right. \\
& \left.+\frac{\sqrt{2} \beta}{\beta-\sqrt{2}} \int_{0}^{1 / 2} \sqrt{1 / 2-s} \frac{3 s^{2}}{2 \sqrt{1-s}} d s\right] \\
& <\frac{1}{\Gamma(3 / 2)} \cdot\left[\left(\frac{\beta}{\beta-\sqrt{2}}\right) \cdot \frac{1}{2}+\frac{\sqrt{2} \beta}{\beta-\sqrt{2}} \cdot \frac{1}{16}\right]<\frac{1}{\Gamma(3 / 2)} \cdot \frac{17}{20}<1 .
\end{aligned}
$$

Then, by Theorem 3.2, we know (4.1) has a nontrivial solution $y^{*} \in C[0,1]$.

EXAMPLE 4.2. Consider the following third-order boundary value problem:

$$
\left\{\begin{array}{l}
\mathbf{D}^{3 / 2} y(t)=\frac{\sqrt{t} y^{2}}{1+y^{2}}-3 t e^{2}+t^{3}, \quad 0<t<1, \\
y(0)=0, \quad y(1)=\frac{1}{2} y\left(\frac{1}{4}\right)
\end{array}\right.
$$

where $f(t, y)=\sqrt{t} y^{2} /\left(1+y^{2}\right)-3 t e^{2}, p(t)=\sqrt{t} / 2, r(t)=3 t e^{2}$. Set $\kappa=\lambda=2$. Now $|f(t, u)| \leq p(t)|u|+r(t),(t, u) \in[0,1] \times \mathbb{R}$ and

$$
\begin{gathered}
\int_{0}^{1} p(s)^{\lambda} d s=\int_{0}^{1} \frac{s}{4} d s=\frac{1}{8}=0.125 \\
{\left[\frac{\Gamma(\alpha)\left(1-\beta \eta^{\alpha-1}\right)[1+\kappa(\alpha-1)]^{1 / \kappa}}{2-\beta \eta^{\alpha-1}+|\beta| \eta^{1 / \kappa}}\right]^{\lambda}=\left[\frac{\Gamma(3 / 2)\left(1-\frac{1}{4}\right) \sqrt{2}}{2-\frac{1}{4}+\frac{1}{4}}\right]^{2}=\frac{9 \pi}{128} \approx 0.2208 .}
\end{gathered}
$$


Therefore

$$
\int_{0}^{1} p(s)^{\lambda} d s<\left[\frac{\Gamma(\alpha)(1-\beta \eta)[1+\kappa(\alpha-1)]^{1 / \kappa}}{2-\beta \eta+|\beta| \eta^{1 / \kappa}}\right]^{\lambda} .
$$

Then, by Theorem 3.3(1), (4.2) has a unique nontrivial solution $y^{*} \in C[0,1]$.

\section{References}

[1] O. P. Agrawal, 'Formulation of Euler-Larange equations for fractional variational problems', J. Math. Anal. Appl. 272 (2002), 368-379.

[2] Z. Bai and H. Lü, 'Positive solutions for boundary-value problem of nonlinear fractional differential equation', J. Math. Anal. Appl. 311 (2005), 495-505.

[3] K. Deimling, Nonlinear Functional Analysis (Springer, Berlin, 1985).

[4] D. Delbosco and L. Rodino, 'Existence and uniqueness for a nonlinear fractional differential equation', J. Math. Appl. 204 (1996), 609-625.

[5] E. R. Kaufmann and E. Mboumi, 'Positive solutions of a boundary value problem for a nonlinear fractional differential equations', Electron. J. Qual. Theory Differ. Equ. (3) (2008), 1-11.

[6] A. A. Kilbas and J. J. Trujillo, 'Differential equations of fractional order: methods, results and problems II', Appl. Anal. 81 (2002), 435-493.

[7] B. Liu, 'Positive solutions of a nonlinear three-point boundary value problem', Comput. Math. Appl. 44 (2002), 201-211.

[8] A. M. Nakhushev, 'The Sturm-Liouville problem for a second order ordinary differential equation with fractional derivatives in the lower terms', Dokl. Akad. Nauk SSSR 234 (1977), 308-311.

[9] I. Podlubny, Fractional Differential Equations, Mathematics in Science and Engineering, 198 (Academic Press, San Diego, 1999).

[10] S. G. Samko, A. A. Kilbas and O. I. Marichev, Fractional Integral And Derivatives (Theory and Applications) (Gordon and Breach, Yverdon, 1993).

[11] S-Q. Zhang, 'The existence of a positive solution for a nonlinear fractional differential equation', J. Math. Anal. Appl. 252 (2000), 804-812.

[12] equations', J. Math. Anal. Appl. 278(1) (2003), 136-148.

YINGXIN GUO, Department of Mathematics, Qufu Normal University, Qufu, Shandong 273165, PR China

e-mail: yxguo312@163.com 